\title{
Using LCA and EPD in Public Procurement Within the Construction Sector
}

\author{
Kristian Jelse and Kristof Peerens
}

\begin{abstract}
Life cycle assessments (LCAs) and Environmental Product Declarations (EPDs) have long been pointed to as tools to be used in green public procurement (GPP), but doing so in practice is challenging. How can such information be requested without breaking some of the general principles for public procurement, for example non-discrimination and transparency? This session was meant to give insights from different stakeholders through five presentations providing different perspectives on this issue. The individual presentations were followed up on by a panel discussion.
\end{abstract}

\section{Introduction}

With the European Union being responsible for more than a third of global resource consumption, $40 \%$ of global energy consumption, $36 \%$ of global carbon dioxide emissions, and one third of global waste streams [1], there is an increasing need for this region to take a leading position when it comes to reducing the overall environmental impact.

Specifically for the construction sector, energy efficient housing is one of the key elements in being able to reduce Europe's overall environmental impact, with studies showing that the embodied energy in construction represents the amount of energy equivalent to more than 75 years of heating [1]. The construction sector overall is more than just housing, and each of the different components have a significant impact on the environment. A rough estimate by the Norwegian authorities has shown that the yearly carbon footprint of the public road system (including construction and maintenance)

K. Jelse $(\bowtie)$

EPD International AB, Stockholm, Sweden

e-mail: kristian@environdec.com

K. Peerens

3 M Belgium bvba/sprl, Diegem, Belgium

(C) The Author(s) 2018

E. Benetto et al. (eds.), Designing Sustainable Technologies,

Products and Policies, https://doi.org/10.1007/978-3-319-66981-6_55 
equates to $\sim 2.3$ million tons of carbon dioxide equivalents, excluding direct traffic emissions (which add 20-25\%) [2].

\section{Challenges in Using LCAs and EPDs as a Standard Assessment Method in Green Public Procurement}

Even though LCA and EPD data is commonly used to quantify the potential environmental impact of construction elements or construction works, reality shows that in GPP, despite environmental considerations being implemented in a variety of European Member States, this data is not used in order to come to conclusions and make decisions related to product or supplier selection [3]. The presentations at the session showed different challenges to different types of stakeholder groups.

\subsection{Challenges for Industry}

LCA is a time and resource intensive undertaking for industry, which requires significant effort in gathering data, creating life cycle models in dedicated software packages, appropriately interpreting the results, and reporting the information in an understandable format. This means that for small and medium sized enterprises, cost is often seen as a barrier to starting such studies [1]. As a result, those companies that have limited resources available cannot always justify the cost, and as a result will not have LCA or EPD data available.

In addition, there are a number of challenges related to communicating LCA and EPD information: some of the information must be specific to the product application and use, other data is relevant for different types of audiences (general public versus architects or project developers). Unfortunately, even when only considering the European Union, means of communication, as well as the different LCA and EPD elements differ [1]. When publishing LCA and EPD data for a number of different countries, there may be a need to generate multiple LCA reports or EPDs, have them verified multiple times (in line with the countries' requirements), and often have them registered locally, each of which comes at a cost. Harmonisation of results can therefore be seen as an absolute must when it comes to promoting the consistent use of LCAs and EPDs [1].

\subsection{Challenges for the Public Sector}

No matter how it is approached, LCA is a complex topic that requires a certain skill set to be able to interpret information generated by the study. So even if LCA and EPD data was to be available for all products participating in a public tender, there 
would need to be quite some knowledge required from the procurement departments in order to identify the best possible product for a certain application [3].

Environmental benchmark data is not readily available, which means that this adds a layer of complexity for public authorities for product differentiation and selection. With the risk of duplicating efforts and lack of consistency, public authorities often use national/regional expertise in order to determine GPP criteria, which can result in different regulatory frameworks, and a lack of harmonisation.

For public authorities, sustainability criteria must go beyond environmental impacts, and must include social, humanitarian and economic aspects as well. Weighting and characterisation of environmental criteria based on the significance of the differences in environmental impacts are therefore a must [4].

\section{Tool and Platform Development to Assist in Interpretation of LCA and EPD Data}

A number of industry sectors, depending on their maturity in the area of sustainability, have built tool sets that help in assessing the overall sustainability impact of their sector's products, technologies and solutions. As a sector, it has therefore been possible to meet the growing demands related to the availability of data. The schemes as developed and deployed in e.g. the steel industry have therefore resulted in collaboration and engagement, increasing transparency and improvements in environmental performance, thus triggering innovation and continuous improvement [4].

Similarly, regional and federal governments (e.g. Germany) as well as building certification schemes are developing databases and software packages that allow for generic and product-specific data to be made available to different stakeholders to allow them to assess the impact of new construction developments or maintenance [5]. Not only have these tools reduced the cost of an LCA, but it ensures that LCA is considered during the construction design and planning [5].

\section{Discussion on Further Efforts to Integrate Data from LCAs and EPDs in GPP}

When considering the different options for integrating LCA and EPD in GPP criteria setting and purchasing decision consistently within any given sector on European level, the key element that must be considered is harmonisation:

- It is a must that schemes operated in different European countries work in the same way and avoid setting national special features in addition to the standards to allow companies to consistently report LCA and EPD data; 
- Benchmarks must be made available in order to ensure that products that are regarded as true differentiators are considered as such regardless of the country they are sold into;

- GPP award criteria must be harmonised, in order to ensure there is a consistent message from the public sector on what the focus areas are supposed to be, and these should be driven by the European Commission;

- For those organisations that are not in a position to generate LCA data themselves, financial support or publicly available data repositories should be made available, driven by the public sector.

Truly implementing science-based product or service selection criteria for purchasing materials or services is the single most important element that will drive a true reduction of the environmental impact of the European construction sector. Based on today's assessment methodologies and data availability, LCAs and EPDs are a critical element in this approach, and should therefore be much more visible in public and private efforts to drive down the environmental impact.

\section{References}

1. Adibi N, Pasquet V, Darul M, Roy A, Salamon A, Challenges to mainstream the use of EPDs in construction Public Procurement in European context, LCM 2017 conference, Luxembourg, 2017.

2. Fredriksen K-S, O'Born R, Implementing climate emission demands in road construction contracts: Insights from the KRAKK project and the Norwegian Public Roads Administration, LCM 2017 conference, Luxembourg, 2017.

3. Ganassali S, Lavagna M, Campioli A, Saporetti S, Green Public Procurement and Construction Sector: EPD and LCA based benchmarks of the whole-building, LCM 2017 conference, Luxembourg, 2017.

4. Brankley L, Tugrul A, Anderson J, Knight D, A Manufacturing Sector's Experience of integrating EPD alongside product, management system and responsible sourcing certification for public procurement, LCM 2017 conference, Luxembourg, 2017.

5. Kusche O, Brockmann T, Figl H, How LCA and EPD are used for implementing Green Public Procurement in the construction sector in Germany, LCM 2017 conference, Luxembourg, 2017.

Open Access This chapter is licensed under the terms of the Creative Commons Attribution 4.0 International License (http://creativecommons.org/licenses/by/4.0/), which permits use, sharing, adaptation, distribution and reproduction in any medium or format, as long as you give appropriate credit to the original author(s) and the source, provide a link to the Creative Commons license and indicate if changes were made.

The images or other third party material in this chapter are included in the chapter's Creative Commons license, unless indicated otherwise in a credit line to the material. If material is not included in the chapter's Creative Commons license and your intended use is not permitted by statutory regulation or exceeds the permitted use, you will need to obtain permission directly from the copyright holder. 